\title{
SISTEMA PARA LA VISUALIZACIÓN EN MAPAS DE INDICADORES EPIDEMIOLÓGICOS DE TUBERCULOSIS PARA la SEcretaría de SALUd dE Manizales
}

Gloria Katherine Mancera-Arango ${ }^{1}$

${ }^{1}$ Magíster en Gestión y Desarrollo de Proyectos de Software. Analista, Bancolombia. Correo electrónico: gmancera@bancolombia.com.co

Resumen. Introducción: el artículo es producto de la investigación realizada en la Universidad Autónoma de Manizales para la Secretaría de Salud Pública de Manizales en el 2015, con el objetivo de obtener indicadores epidemiológicos para la enfermedad de tuberculosis de forma rápida y confiable. Además, de incluir elementos de georreferenciación con el objetivo de aportar en la toma de decisiones de la unidad. Metodología: para el desarrollo del trabajo se eligió Openup, un proceso unificado que aplica enfoques iterativos e incrementales dentro de un ciclo de vida estructurado, y se llevaron a cabo tres etapas: inicio del proyecto, desarrollo de la aplicación y despliegue y validación con la entidad. Resultados: el sistema desarrollado permite obtener indicadores epidemiológicos de la enfermedad de tuberculosis en un gráfico de barras (como, por ejemplo, la cantidad de personas afectadas por comuna), lo que permite relacionar la información con las variables socioeconómicas elegidas; también permite realizar la migración de los archivos de Sivigila, Sisben y Estadística, los cuales contienen la información necesaria para realizar la consulta y la posterior visualización de la información en los gráficos y en un mapa, lo que lo convierte en una herramienta web. Conclusiones: con el proyecto se brindó una herramienta de fácil acceso para los usuarios de la Secretaría de Salud de Manizales, logrando obtener información de forma ágil de los indicadores seleccionados, y disminuyendo los tiempos en la construcción de los mismos.

Palabras claves: indicadores, software, tuberculosis, vigilancia epidemiológica, visualización georreferenciada. 


\title{
SYSTEM FOR VIEWING EPIDEMIOLOGICAL INDICATORS OF TUBERCULOSIS ON MAPS FOR THE Manizales Health Secretary's Office
}

\begin{abstract}
Introduction: The article arises from the research conducted in 2015 at the Universidad Autónoma de Manizales for the Manizales Public Health Secretary's Office, its purpose was to obtain epidemiological indicators of tuberculosis rapidly and reliably and contribute to the unit's decision making by including georeferencing elements. Method: Openup, a unified process that applies iterative and incremental approaches within a structured lifecycle, was chosen for the task, and three stages were carried out: project startup, application development, and deployment and validation with the entity. Results: The system developed allows to obtain epidemiological indicators of tuberculosis disease in a bar graph (such as the number of people affected by district), which helps to match information with chosen socioeconomic variables. It also enables the migration of sivigiLA, SISBEN and Estadística files, which contain the information necessary to query and view the information in graphs and on maps, making it a web tool. Conclusions: The project provided an easily accessible tool for users at the Manizales Health Secretary's Office to obtain information of the selected indicators and reduce the time for their construction.
\end{abstract}

Keywords: indicators, software, tuberculosis, epidemiological surveillance, georeferenced view.

\section{SISTEMA PARA A VISUALIZAÇÃO DE INDICADORES EPIDEMIOLÓGICOS DE TUBERCULOSE EM MAPAS PARA A Secretaria da Saúde de Manizales (Colômbia)}

Resumo. Introdução: este artigo é produto da pesquisa realizada na Universidad Autónoma de Manizales para a Secretaria da Saúde Pública de Manizales, em 2015, com o objetivo de obter indicadores epidemiológicos para a doença de tuberculose de forma rápida e confiável. Além disso, incluir elementos de georreferenciação a fim de contribuir para a tomada de decisões da unidade. Metodologia: para o desenvolvimento do trabalho, foi escolhido o Openup, um processo unificado que aplica abordagens iterativas e incrementais dentro de um ciclo de vida estruturado; além disso, foram realizadas três etapas: início do projeto, desenvolvimento da aplicação e implantação, e validação com a entidade. Resultados: o sistema desenvolvido permite obter indicadores epidemiológicos da tuberculose num gráfico de barras (por exemplo, a quantidade de pessoas afetadas por comunidade), o que permite relacionar a informação com as variáveis socioeconômicas escolhidas; também realizar a migração dos arquivos de Sivigila, SisBen e Estatística, os quais contêm a informação necessária para realizar a consulta e a posterior visualização da informação nos gráficos e num mapa, o que o converte numa ferramenta web. Conclusões: com o projeto, ofereceu-se uma ferramenta de fácil acesso para os usuários da Secretaria da Saúde de Manizales, obtendo informação dos indicadores selecionados de forma ágil e diminuindo os tempos na construção destes.

Palavras-chaves: indicadores, software, tuberculose, vigilância epidemiológica, visualização georreferenciada. 


\section{Introducción}

En el mundo existe una problemática con respecto a las enfermedades que son catalogadas como epidemiológicas. Las personas pueden ser vulnerables a contraer dichas enfermedades, si no se tiene conocimiento de la causa, el origen o el lugar de concentración; asimismo, las desigualdades socioeconómicas ayudan a potenciar la vulnerabilidad de contagio de la población. Para los gobiernos, dicho conocimiento es importante ya que pueden atacar el problema con campañas de prevención, educación y mitigación, al igual que encontrar la forma de destinar recursos a las regiones más vulnerables y, por lo tanto, concentrar esfuerzos donde más se necesitan.

En este trabajo se desarrolló una solución tecnológica que permite a la Secretaría de Salud conocer de forma rápida los valores de los indicadores, la concentración depersonasyelestado socioeconómico de los afectados por la enfermedad de tuberculosis en Manizales. A lo largo del trabajo se analizaron herramientas tecnológicas libres adecuadas que permiten desarrollar el proyecto, con las cuales se puede entregar de forma idónea la información para la toma de decisiones.

\section{2. Área problemática}

Con el fin de entrar en materia acerca del problema a tratar en el presente trabajo, se debe hablar primero que todo de la epidemiología, disciplina definida por la Organización Mundial de la Salud como el "estudio de la distribución y los determinantes de estados o eventos (en particular de enfermedades) relacionados con la salud y la aplicación de esos estudios al control de enfermedades y otros problemas de salud" [1].

Las enfermedades epidemiológicas entonces, según lo anterior, son alteraciones en la salud que afectan de manera parcial o total a una determinada población, para este caso, la población de Manizales, quienes son objeto de estudio para el cumplimiento del fin del presente trabajo.

Como se consagra en la Constitución Política de Colombia, en el artículo 49: "La atención de la salud y el saneamiento ambiental son servicios públicos a cargo del Estado. Se garantiza a todas las personas el acceso a los servicios de promoción, protección y recuperación de la salud. Corresponde al Estado organizar, dirigir y reglamentar la prestación de servicios de salud a los habitantes y de saneamiento ambiental conforme a los principios de eficiencia, universalidad y solidaridad" [2].

Con lo anterior, las entidades gubernamentales están en la obligación de velar porque el derecho a la salud sea cumplido; a nivel municipal, la entidad encargada de velar por dicho objetivo es la Secretaría de Salud Pública de la Alcaldía de Manizales (SSPM), con el objetivo de liderar y desarrollar programas, proyectos y políticas en el desarrollo del Sistema General de Seguridad Social en Salud del Municipio de Manizales acorde con la normatividad nacional, buscando mejorar la calidad de vida de los habitantes desde el componente de salud en los próximos años [3].

LA SSPM se compone de varias unidades; cada una de las cuales se enfoca en un objetivo específico que contribuye a la obtención de los resultados propuestos por la secretaría. Entre las unidades se encuentran:

- Planeación y Epidemiología

- Prestación y Desarrollo de Servicio de Salud

- Salud Pública

- Área Financiera

- Área Jurídica

Para la unidad de Planeación y Epidemiología, es importante: "El monitoreo del estado de salud, enfermedad, indicadores y los eventos de interés en salud pública en el municipio, con el objetivo de obtener información para desarrollar proyectos estratégicos que impacten a la comunidad de acuerdo a lo que se está presentando en su momento" [4].

El Instituto Nacional de Salud, a su vez, utiliza los protocolos que la Organización Panamericana de Salud estipula, con el fin de articular los datos a nivel departamental, nacional, y en Suramérica.

El Instituto Nacional de Salud [5], a través del programa Sivigila, propone los indicadores para cada tipo de evento epidemiológico, ya que cada enfermedad se manifiesta y afecta de forma diferente a la comunidad. Según el manual de Sivigila, los indicadores "permiten medir de forma cuantitativa o cualitativa, sucesos colectivos con el fin de sustentar las gestiones políticas y medir los logros y metas".

Estos índices estadísticos ayudan a una entidad a conocer el estado de salud de una población específica, mediante la recolección de información 
emitida en un censo o encuesta. Estos indicadores pueden ser cualitativos o cuantitativos, siendo el primero una medida de cantidad o, en este caso, el número de personas afectadas por cierta enfermedad en una zona específica. Los indicadores cualitativos son opiniones o percepciones que tiene la población acerca de un tema de salud específico. Estos permiten tener un registro de apariciones y distribuciones de una enfermedad en un grupo de personas determinado, ayudando a tomar decisiones para mitigar las alteraciones en la salud.

Como se observa en la página oficial del Departamento Nacional de Planeación (DNP) en el Sisben [6], se reporta la información socioeconómica de la población, como, por ejemplo, el estrato de la vivienda o el nivel educativo, entre otras, permitiendo al DNP identificar y clasificar a las personas que no pueden cubrir sus necesidades básicas para que el estado pueda suplirles los derechos fundamentales, otorgando subsidios que les permiten ser beneficiarios de diferentes programas.

Sivigila es un programa sugerido por el Instituto Nacional de Salud (INS) a todas las entidades departamentales, distritales o municipales de salud. De acuerdo con lo anteriormente mencionado, se evidencia la importancia que tiene para el gobierno la vigilancia de la salud pública y los registros de las enfermedades; específicamente, la Secretaría de Salud de Manizales, como entidad municipal, debe cumplir con los requerimientos y registrar la información de los diferentes eventos que se presentan en la ciudad.

En el manual del programa Sivigila [5] se encuentran los indicadores que se deben analizar en los diferentes eventos, clasificándolos como:

- De acuerdo con el momento de su aplicación, posee tres tipos de indicadores que son:

- Indicadores de estructura: indicadores estandarizados, son la base de la planeación.

- Indicadores de coyuntura: son los referentes del seguimiento y control de un plan de desarrollo de un programa gubernamental.

- Indicadores de referencia: son los que se utilizan para los datos socioeconómicos y demográficos.
- Indicadores de proceso: permiten evaluar la gestión de la entidad con respecto a la vigilancia, actividades realizadas para alcanzar determinado resultado.

- Indicadores de resultado.

- Indicadores de impacto: se refiere al producto final, permite medir efectos a mediano y largo plazo generados por una política, programa o proyecto sobre la población en general.

De los anteriores tipos de indicadores, en el presente trabajo se utilizaron los indicadores de proceso y de impacto, que son los más prioritarios para la Secretaría de Salud, y por lo tanto, la herramienta que se desarrolló solo acoge estos para la enfermedad de tuberculosis y tuberculosis extrapulmonar.

Una variable, en nuestro caso, es una propiedad que tiene relación con las personas que pertenecen a un grupo poblacional afectado por la tuberculosis. La Secretaría de Salud desea conocer el comportamiento de tres variables en la comunidad, visualizándolas en un mapa con el objetivo de hacer un análisis para la gestión de control del evento; sus categorías son:

- Género: conocer la cantidad de personas por sexo (masculino/femenino) que enferman por comuna y el porcentaje que representa.

- Edad: en un rango de edad conocer la cantidad de personas que enferman y el porcentaje que representa.

- Tipo de tuberculosis: conocer la cantidad de personas que enferman por tipo de tuberculosis por cada comuna.

\section{Antecedentes}

Para una mejor comprensión acerca del tema propuesto en la tesis, fue necesario realizar una investigación previa que ayudó a identificar las mejores herramientas y tecnologías que sirvieron de apoyo para crear la solución planteada.

- De acuerdo con el aspecto evaluado: 
Como primer trabajo de investigación, se encontró que la Organización Panamericana de la Salud [7] desarrolla una aplicación denominada SIG-EPI. Esta tiene el objetivo de obtener información (alfanumérica y georreferenciada), mediante unas herramientas específicas, contando con diferentes módulos para la vigilancia de la salud pública y la epidemiología. Algunos de estos módulos identifican, por ejemplo, patrones regionales en el Análisis de Situación de Salud (AsIs), vigilancia y monitoreo de salud pública, ubicación de casos de enfermedades (georreferenciación), y evaluación de impacto de intervenciones poblacionales, entre otros.

Otro trabajo investigado es el Epidat [8], un programa de libre distribución desarrollado por el Servizo de Epidemioloxía de la Dirección Xeral de Innovación e Xestión da Saúde Pública de la Consellería de Sanidade (Xunta de Galicia), con el apoyo de la Organización Panamericana de la Salud (ops-OMs) y la Universidad CES de Colombia. Dentro de los objetivos de Epidat, se encuentran:

- Aportar una herramienta de manejo sencillo y de utilidad para el análisis estadístico y epidemiológico de datos que cubra algunas lagunas en las aplicaciones más extendidas.

- Apoyar y facilitar la docencia en epidemiología, tanto en el ámbito de la cooperación técnica, como en el universitario, y así fortalecer la capacidad analítica de los profesionales de salud.

Otro trabajo investigado es el Software Centers for Disease Control and Prevention, en Atlanta, Georgia, Estados Unidos, realizado en el 2014 [9]. Este trabajo posee un conjunto de herramientas que permiten la creación de instrumentos de recolección y análisis de datos, su visualización y presentación de informes, utilizando métodos estadísticos que permiten un análisis avanzado y mapeo de datos. Este software está presente en más de 181 países y traducido a 13 idiomas. Entre sus principales funciones se encuentran:

- Creación de cuestionarios

- Creación de bases de datos

- Personalización del proceso de entrada de datos llamado MakeView

- Programas para realizar la entrada de datos a los cuestionarios, denominados Enter y EpiMap, los cuales permiten la creación de mapas de SIG sobrepuestos con datos de la encuesta

Otra herramienta interesante que se investigó es el Sistema Gráfico Georreferenciado de Indicadores de Género, desarrollado en Ciudad de México en el 2010 [10]. Este permite la representación de indicadores de género en los estados o municipios, y "tiene como característica representar geográficamente la información, relacionándola con zonas o regiones culturalmente diferenciadas mediante consultas de mapas temáticos de indicadores sobre población, pobreza, educación, salud, violencia, entre otros" [11].

A fin de complementar la investigación, se agrega el trabajo de la autora Luz Constanza Gatica, denominado "Análisis de distribución de patrones territoriales en casos de tuberculosis", desarrollado en Santiago de Chile en el 2014 [12]. En este trabajo se describe cómo la tuberculosis afecta 32 comunas de Santiago de Chile, con el objetivo de identificar la distribución de los casos de tuberculosis y la asociación de estos con algunas variables a la agrupación de los casos y los determinantes sociales. Todo lo anterior se presenta mediante procesos de análisis de estadística espacial de patrones, como, por ejemplo, la técnica del vecino más cercano. El software de sIG utilizado en el trabajo de Gatica fue ArcGis 9.3 (software licenciado).

De acuerdo con los trabajos anteriormente mencionados, se resalta el uso de las tecnologías como apoyo importante a la georreferenciación de un problema en común para una comunidad específica. El conocimiento del problema ayuda a acotar la función que debe cumplir el sistema que se plantea, al igual que la división de este para atacar de forma concreta las zonas de mayor riesgo. En la tesis de grado se toma el uso de la georreferenciación para ubicar las zonas de Manizales donde se presente la enfermedad de la tuberculosis (indicador objeto del trabajo), al igual que la representación de los datos obtenidos por las entidades de salud, los cuales sirven para alimentar la base de datos del sistema y así concluir en algún programa que mitigue esta enfermedad.

Por último, se puede decir que los trabajos investigados permiten afianzar la tesis acerca de los sistemas de información como herramientas de apoyo para georreferenciar variables e identificar problemáticas que afectan a una población. También, como problemáticas encontradas 
aparecen los factores sociales y las desigualdades económicas, las cuales influyen fuertemente en la salud de la población, permitiendo la propagación de una enfermedad epidemiológica. La creación de una herramienta de este tipo (sistema de información georreferenciado), puede llegar a hacer aportes importantes a una entidad pública, al permitirle aprovechar más la información que tiene en sus bases de datos.

\section{Referente teórico}

La epidemiología, según los autores MacMahon y Pugh [13], es el estudio de la distribución, los determinantes y de la frecuencia de una enfermedad en el ser humano. Por otro lado, el autor Lilienfild [13] dice que la epidemiologia es el estudio de la distribución de una enfermedad o una condición en una población, así como de aquellos factores que influyen en su distribución.

La vigilancia epidemiológica es una de las bases de la salud pública, encargada de velar por la comunidad en general, ya que busca su bienestar, siendo el estado el responsable de ello. Esta se encuentra íntimamente relacionada con el desarrollo social (condiciones económicas, políticas, sociales y científicas).

La tuberculosis (тв) es una infección producida por el Mycobacterium tuberculosis hominis, también llamado bacilo de Koch (вк), en honor a su descubridor. Es una enfermedad de localización pulmonar, que no solo afecta al pulmón propiamente, sino también a los ganglios hiliares vecinos y a los bronquios. Afecta también a otros órganos, como el cerebro, las meninges, los huesos, el hígado, los riñones y la piel (тв extrapulmonar).

Según el Ministerio de Salud y Seguridad Social, la tuberculosis se trasmite de persona a persona por el aire; cuando es infectada con $\mathrm{TB}$, esta estornuda y expulsa el $\mathrm{BK}$, con lo cual las personas cercanas quedan expuestas a contraer la enfermedad; a lo largo de un año, un enfermo de тв puede infectar a 10 o 15 personas de contacto permanente, si este no recibe el tratamiento adecuado [14].

Como afirma la Organización Mundial de la Salud (OMs), los determinantes sociales de salud afectan a un individuo desde que nace, mientras crece, trabaja y envejece, como resultado de la distribución de los recursos sociales y económicos (dinero, poder, condiciones de vida y de salud), afectando de forma positiva o negativa a las personas con respecto a la salud. Estos determinantes fueron estudiados y evaluados por la oms, concluyendo que cada vez más afectan de forma negativa a la población más vulnerable, y estipuló unos lineamientos para los gobiernos con el propósito de mitigar el impacto que están presentado en la población. Estos lineamientos fueron creados en el 2005 y el informe final se entregó en el 2008.

La oms diseñó un manual en el que propone implementar medidas que ayudan a mejorar las condiciones de vida de una población, y así lograr disminuir la cantidad de personas afectadas por enfermedades [15]. Según el autor Manuel Villar-Aguirre [16], los determinantes de la salud han estado presentes en la historia de la humanidad, siendo de gran influencia en el estado de la salud de la población. Los factores como la pobreza, las malas condiciones de vida, así como el estado medio ambiental y económico, influyen, tanto en la morbilidad, como en la mortalidad de los individuos. Villar-Aguirre afirma que, mediante estudios realizados en años anteriores, se determinó que el Estado es el responsable de velar por la salud, y a partir de dichos estudios nacieron los factores determinantes de la salud pública, buscando con ello disminuir el impacto en la población de dichos factores.

Luz Stella Álvarez-Castaño [17] trabaja sobre los determinantes sociales de salud y los factores de riesgo, y expone cómo dichos factores no son lo mismo que los determinantes de salud, siendo estos últimos los que afectan a un grupo grande de la población, mientras que los factores de riesgo son los que afectan a nivel individual.

Para la Secretaría de Salud de Manizales, los factores determinantes de la salud juegan un papel importante en el análisis y la toma de decisiones con respecto a las condiciones de salud de la ciudad, así como para conocer la presencia de dichos determinantes en la cuidad y la relación con los indicadores de тв.

Los determinantes sociales de salud se pueden encontrar registrados en la bases de datos del Sisben, ya que allí está la información social, económica y las condiciones de vida en que están los colombianos encuestados, y sirve como base en el análisis y la toma de decisiones del gobierno. 


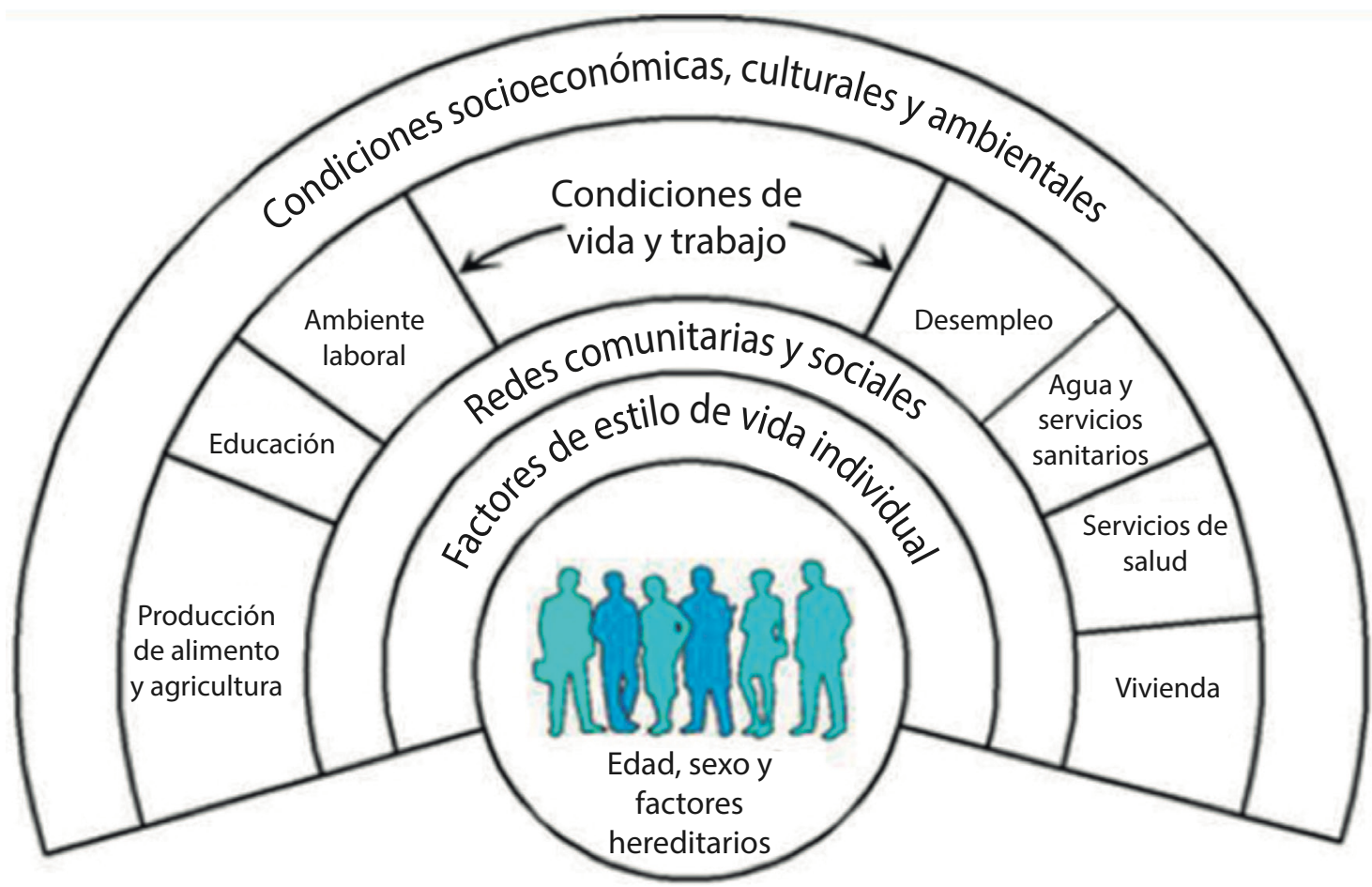

Figura 1. Determinantes de la salud, ilustrados en Determinantes Sociales de Salud (DSs) y Estrategias Políticas de Acción, trabajo realizado por integrantes de la oms

Fuente: [18]

Según Peña-Llopis [19], un sistema de información geográfica (sIG) es una herramienta que procesa datos y mapas digitalizados, con el fin de analizar y visualizar información geográfica de forma rápida para el estudio y la toma de decisiones por parte de los usuarios del mismo.

Un sig se compone de procesos, métodos, instrumentos y datos, permitiendo capturar, almacenar, procesar y presentar al usuario la información final de forma alfanumérica y georreferenciada.

Según la página especializada en temas SIG [21], la georreferenciación es la asignación de coordenadas de mapa y ubicación espacial a objetos cartográficos. Estos, a su vez, están compuestos por ubicación geográfica real y extensión específica de las posiciones de la tierra; la correcta ubicación es necesaria, tanto a nivel de cartografía básica (mapas en papel), como para los sIG.

En este proyecto se utilizólageorreferenciación para cada comuna, lo que permite la visualización de Manizales en un mapa digital. Asimismo, identificar la cantidad de personas afectadas por esta enfermedad con indicadores epidemiológicos o filtros de género, edad o tipo de tuberculosis.

\section{Pregunta de investigación}

¿Cómo puede la Secretaría de Salud de Manizales consultar los indicadores epidemiológicos de tuberculosis que relacionen los datos proporcionados por el Sivigila y el Sisben, con el propósito de apoyar la vigilancia epidemiológica de la enfermedad al visualizar su presencia en las comunas de la ciudad?

\section{Justificación}

Gracias a la globalización y a las tecnologías de la información, las organizaciones actualmente tienen la posibilidad de administrar mejor y obtener datos más confiables y oportunos. Esto les permite tomar decisiones de manera más rápida y acertada. 


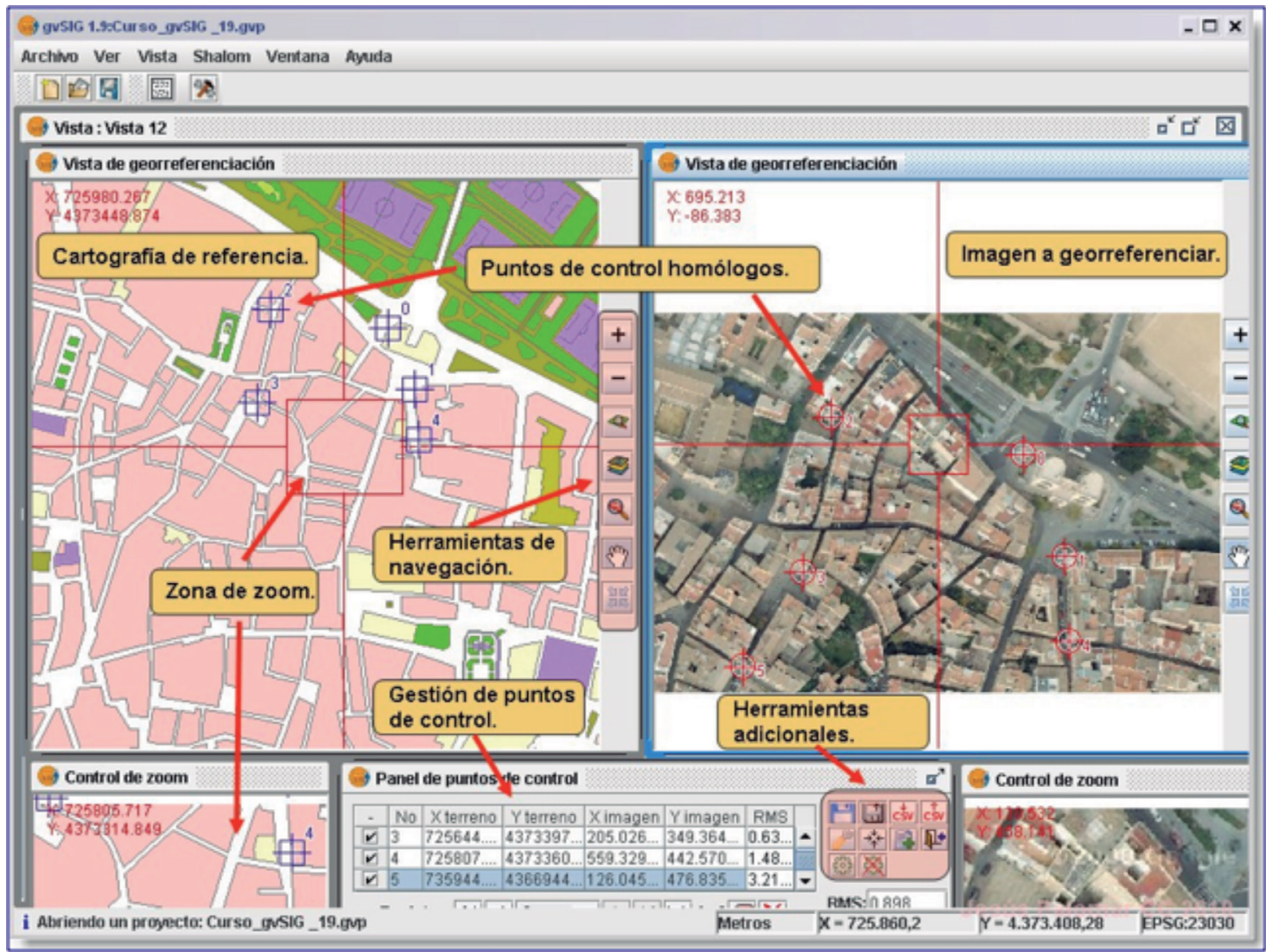

Figura 2. Georreferenciación realizada e ilustrada en el programa gvsig Fuente: [20]

Las entidades que ejercen control y vigilancia en el área de salud no son ajenas a esta globalización, y día a día emplean herramientas tecnológicas que les permiten agilizar los procesos para interpretar mejor la información contenida en las bases de datos, lo cual es fundamental en la toma de decisiones gerenciales, y así adaptar nuevas estrategias a los diferentes programas de gestión de la salud.

Se realizó un análisis de las tecnologías y procesos que actualmente se manejan en la SSPM, y se encontró que estos no son tan eficientes como se esperaba. Ocupan personal, esfuerzos y tiempo que podrían dedicarse a otras labores igual de importantes, y así cumplir con su objetivo en menos tiempo.

El sistema de indicadores será una herramienta estratégica para la entidad, porque permitirá relacionar la información de salud y la socioeconómica por medio de variables de importancia identificadas por el epidemiólogo, con el fin de realizar el análisis de los determinantes de salud. El no contar con dichos elementos lleva a desaprovechar el potencial de la información, analizando el estado de cada una de las personas, pero agrupadas por zonas estratificadas de la ciudad. Con la georreferenciación el usuario visualizará la información de los indicadores en un mapa y llegará a una mejor compresión, convirtiéndose en una buena herramienta de apoyo a la gestión de decisiones en lo que respecta a la salud en Manizales.

\section{Objetivos}

\section{General}

Desarrollar un sistema para la consulta y visualización de indicadores en un mapa digital de Manizales, con gráficos de barras de tres indicadores de la 
enfermedad de tuberculosis y filtros como herramienta de apoyo para la vigilancia epidemiológica en la Secretaría de Salud de Manizales, y datos suministrados por el Sivigila.

\section{Específicos}

- Determinar la información que se debe obtener de los sistemas Sivigila y Sisben, a fin de que se utilice como fuente de alimentación para el Sistema de Indicadores Epidemiológicos Georreferenciados.

- Establecer la arquitectura para el Sistema de Indicadores Epidemiológicos Georreferenciados con base en el resultado de la investigación de antecedentes.

- Construir una plataforma web que permita consultar de forma georreferenciada indicadores epidemiológicos para el evento de la enfermedad de tuberculosis.

- Validar los resultados presentados por el sistema para la visualización del mapa e indicadores epidemiológicos, mediante contrastación con los análisis realizados por la Secretaría de Salud de Manizales para la tuberculosis.

\section{Metodología}

En el desarrollo del presente proyecto, se eligió como guía principal el proceso Openup y umL para los diagramas del sistema; el diseño de la arquitectura se trabajó con vistas $4+1$.

Openup es un proceso de desarrollo de software, el cual permite desarrollar un proyecto o programa con un ciclo de vida iterativo e incremental, presentando avances a los clientes. Para este caso, a la Secretaría de Salud, que recibe entregas iterativas del resultado que pide, y constata así el alcance que toma el software [22].

Se plantearon tres etapas, acordes con las fases propuestas por OpenuP:

- Inicio del proyecto (fase de inicio)

- Desarrollo de la aplicación (fases de elaboración y construcción)

- Despliegue y validación con la entidad (fase de transición)

\section{Inicio del proyecto}

Esta etapa está alineada con la fase de inicio de Openup. El objetivo de esta fase es llevar a cabo el acercamiento entre la Secretaría de Salud y la persona que desarrolla el proyecto, para así obtener la información del problema, los objetivos y determinar si el proyecto es viable. También busca:

- Conocer los procesos de obtención de indicadores.

- Obtener la lista de requerimientos principales.

- Analizar riesgos del desarrollo.

\section{Desarrollo de la aplicación}

Esta etapa comprende las fases de elaboración y construcción del proceso de Openup; cada fase contiene actividades, prácticas y artefactos que permiten el desarrollo del software a través del tiempo.

\section{Despliegue y validación con la entidad}

Esta etapa está alineada con la fase de transición de Openup, cuyo objetivo es asegurar que el software esté listo para ser entregado a los usuarios. En el proyecto el objetivo es entregar al usuario final el sistema para la visualización de mapas, los indicadores de tuberculosis y los indicadores, y así obtener la aprobación del mismo.

A continuación, se presenta una tabla con el resumen del plan de iteraciones realizadas durante la construcción del proyecto.

La tabla 1 muestra los incrementos que se elaboraron en cada iteración. La prioridad y los incrementos son:

- Definición del alcance: se desarrolló el módulo web que contendrá todos los otros módulos planteados.

- Módulo web + Módulo mapa: primero se creó el Módulo mapa y luego se integró con el Módulo web. 
Tabla 1. Plan de iteraciones en la construcción de la aplicación

\begin{tabular}{|c|c|c|c|c|c|c|}
\hline \multirow[t]{2}{*}{ N. ${ }^{\circ}$} & \multirow[t]{2}{*}{ Incremento } & \multirow[t]{2}{*}{ Objetivo } & \multirow[t]{2}{*}{ Actividades } & \multicolumn{3}{|c|}{ Prioridad } \\
\hline & & & & 颉 & 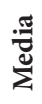 & $\stackrel{\text { ت }}{\frac{\pi}{4}}$ \\
\hline 1 & $\begin{array}{l}\text { Definición del } \\
\text { alcance }\end{array}$ & $\begin{array}{l}\text { Crear módulo web de la } \\
\text { aplicación }\end{array}$ & $\begin{array}{l}\text { Investigar sobre el framework sugerido por } \\
\text { el asesor de la Secretaría de Salud. } \\
\text { Desarrollar los mockups de las posibles } \\
\text { interfaces. } \\
\text { Implementar el módulo de ingreso. } \\
\text { Aplicar diseño hojas de estilo al proyecto. }\end{array}$ & & $\mathrm{X}$ & \\
\hline 2 & $\begin{array}{l}\text { Módulo web + } \\
\text { Módulo mapa }\end{array}$ & $\begin{array}{l}\text { Implementar librería que per- } \\
\text { mita visualizar en un mapa la } \\
\text { información alfanumérica. }\end{array}$ & $\begin{array}{l}\text { Investigar librería que permita implemen- } \\
\text { tar el mapa. } \\
\text { Desarrollar módulo del mapa. } \\
\text { Crear la estructura de base de datos. } \\
\text { Integrar base de datos con módulo mapa. }\end{array}$ & & & $\mathrm{X}$ \\
\hline 3 & $\begin{array}{l}\text { Módulo web + } \\
\text { Módulo mapa }+ \\
\text { Módulo gráficos }\end{array}$ & $\begin{array}{l}\text { Implementar librería que per- } \\
\text { mita visualizar en gráficos de } \\
\text { la información alfanumérica. }\end{array}$ & $\begin{array}{l}\text { Investigar librería que permita implemen- } \\
\text { tar un gráfico para los indicadores. } \\
\text { Desarrollar módulo de gráficos. } \\
\text { Integrar base de datos con el módulo de } \\
\text { gráficos. }\end{array}$ & & & $\mathrm{X}$ \\
\hline 4 & $\begin{array}{l}\text { Modulo web + } \\
\text { Modulo mapa + } \\
\text { Modulo gráficos } \\
+ \text { construcción } \\
\text { de indicadores }\end{array}$ & $\begin{array}{l}\text { Implementar los SQL (con- } \\
\text { sultas) para la consulta de } \\
\text { los diferentes indicadores y } \\
\text { filtros. }\end{array}$ & $\begin{array}{l}\text { Construir SQL para indicadores. } \\
\text { Construir SQL para filtros. }\end{array}$ & & & $\mathrm{X}$ \\
\hline 5 & $\begin{array}{l}\text { Módulo de } \\
\text { migración }\end{array}$ & $\begin{array}{l}\text { Desarrollar el módulo de mi- } \\
\text { gración de la información de } \\
\text { los sistemas, Sisben, Sivigila y } \\
\text { Estadística. }\end{array}$ & $\begin{array}{l}\text { Investigar la librería que permita migrar la } \\
\text { información de Excel a la base de datos. } \\
\text { Implementar librería que permita migrar } \\
\text { de Excel en el módulo de migración. }\end{array}$ & & $\mathrm{X}$ & \\
\hline 6 & $\begin{array}{l}\text { Sistema com- } \\
\text { pleto }\end{array}$ & Entregar el sistema completo. & $\begin{array}{l}\text { Realizar pruebas del sistema con informa- } \\
\text { ción real y comparar con análisis previos } \\
\text { contenidos en el manual. }\end{array}$ & & & $\mathrm{X}$ \\
\hline
\end{tabular}

Fuente: elaboración propia.

- Módulo web + Módulo mapa + Módulo de gráficos: se creó el módulo de gráficos y se integró al Módulo Web.

- Módulo web + Módulo mapa + Módulo gráfico + construcción de consultas: se desarrollan las diferentes consultas del sistema a nivel base de datos.

- Módulo migración: se realiza todo el módulo de migración para los tres tipos de archivos (Sivigila, Sisben y Estadística).
- Se hicieron pruebas a todos los módulos integrados: se corrigieron errores y se subió al servidor de aplicación (hosting).

En la figura 3, se muestran los componentes contenidos en el sistema:

- Cliente. Es el usuario final que accede al sistema para realizar consultas de indicadores, filtros o migración, a través de una plataforma web consultada vía Internet. 


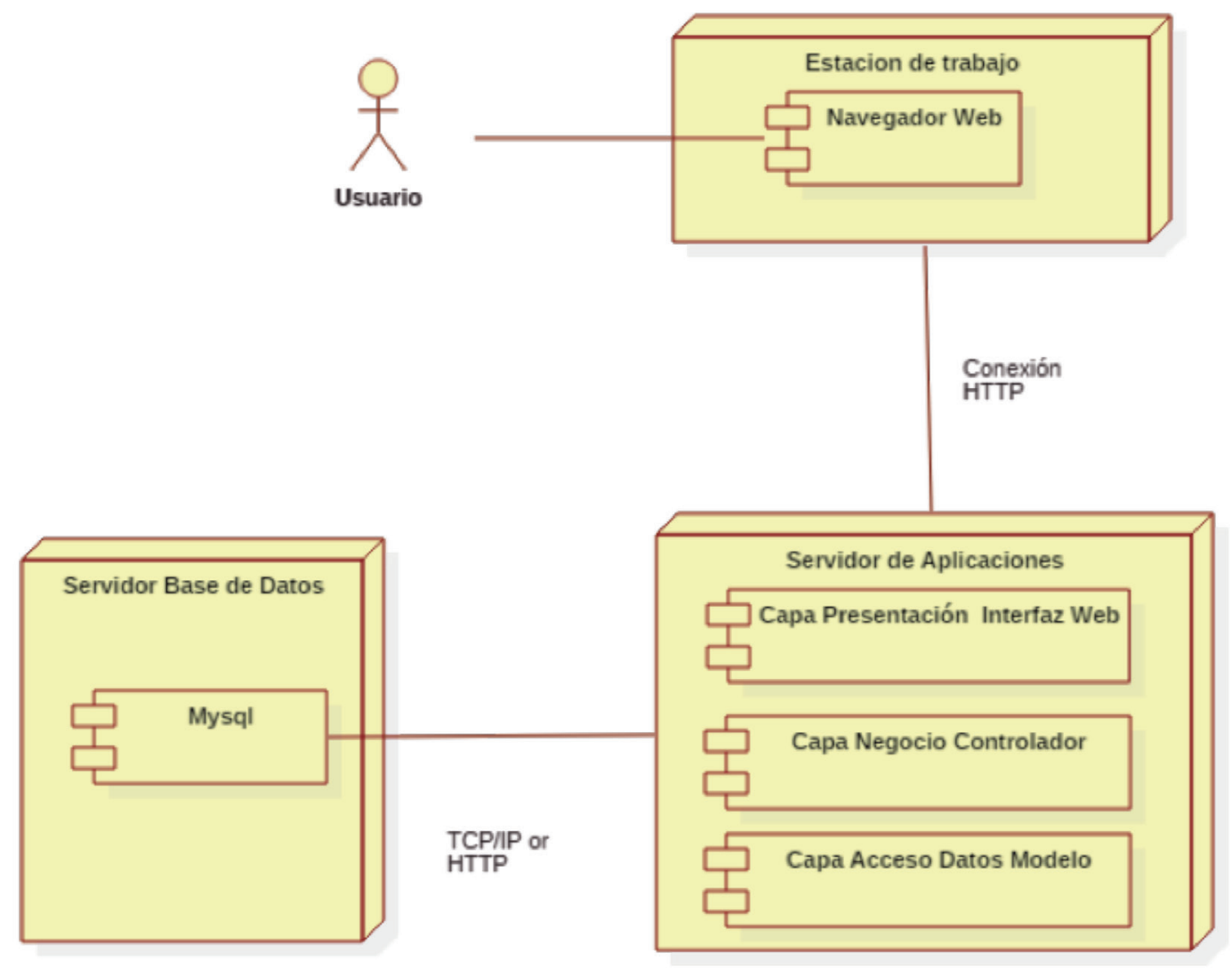

Figura 3. Diagrama de arquitectura del sistema planteado Fuente: elaboración propia

- Capa de presentación. Es la primera capa de la aplicación, la cual se presenta al usuario mediante una interfaz gráfica y es la comunicación con las demás capas del sistema.

- Capa de negocio. Realiza de forma independiente los procesos pertenecientes a la lógica del negocio. Esta lógica realiza el procesamiento de la información que viaja desde la capa de presentación, permitiendo de esta forma actualizar un componente, si es necesario, de la base de datos o de la misma capa de presentación, para luego retornar los datos hacia el usuario.

- Capa de datos. Esta capa es la encargada de realizar las transacciones hacia la base de datos, tales como consultas, actualización de información e inserción de la misma; esta se encuentra alojada en el servidor de aplicaciones, al igual que las dos capas anteriores a ella.
- $\quad M y S Q L$. El motor utilizado para esta aplicación es MYsQL, un sistema de gestión de base de datos que se encarga de almacenar la información consultada, migrada y actualizada por la lógica de negocio. El motor se encuentra instalado en un servidor de base de datos independiente al servidor de aplicaciones.

En la figura 4 la vista de procesos del sistema permite visualizar las opciones:

Migración. En esta opción el sistema visualiza las pantallas para que el usuario realice la migración de los sistemas Sivigila, Sisben y Estadística; el sistema realiza las validaciones necesarias de la acción, que es guardar la información de los archivos en el sistema.

Las validaciones están predefinidas para cada tipo de archivo. En el sistema sería cada opción de importar información de cada sistema; si las 


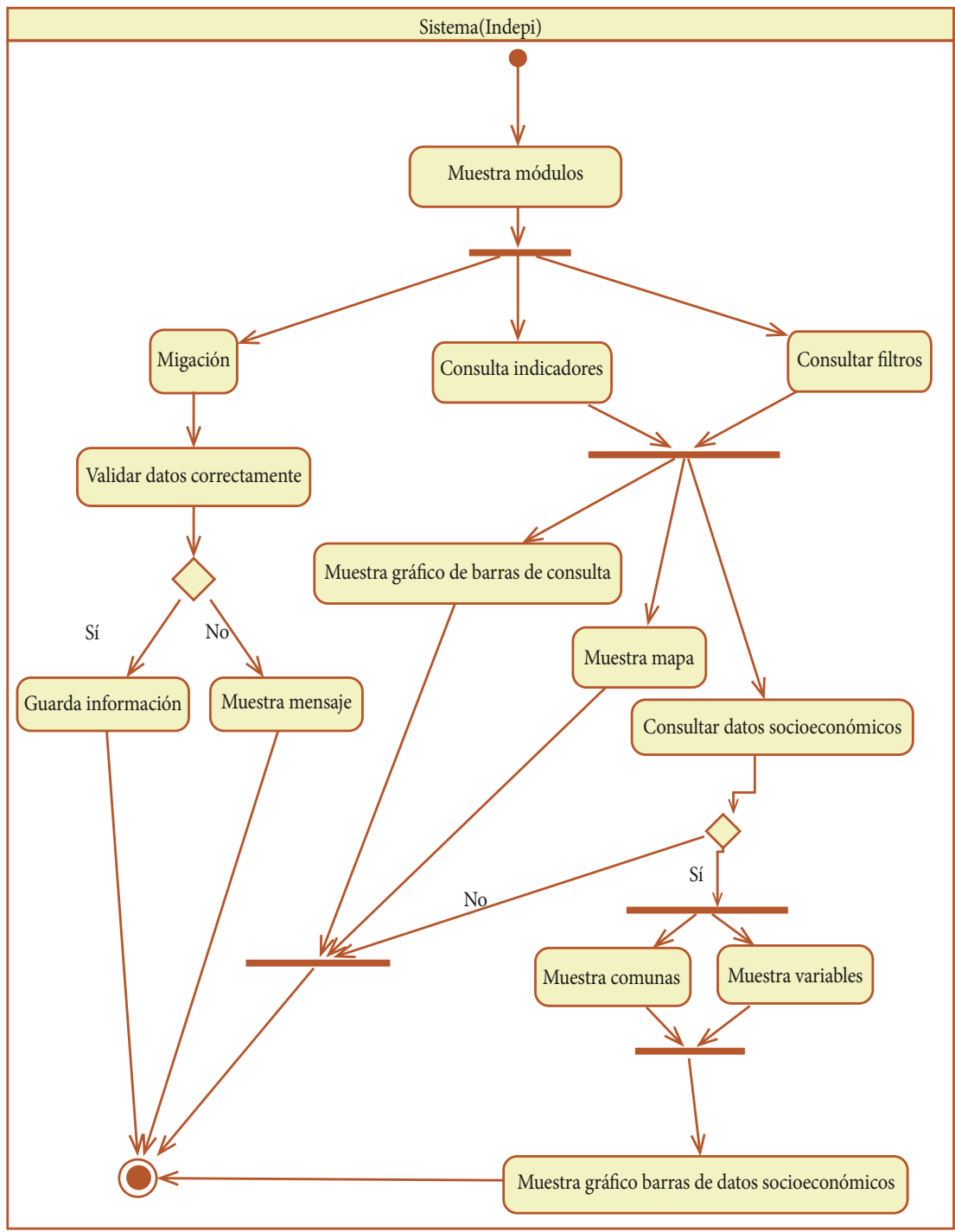

Figura 4. Vista de procesos diagrama de actividades del sistema Fuente: elaboración propia

validaciones son perfectas, el sistema visualiza un mensaje de importación correcta, en el caso contrario presenta un mensaje informando cuál fue la causa de la no importación.

Consulta indicadores y filtros. En esta opción el sistema presenta varias opciones de selección que permiten hacer la consulta de los indicadores o filtros; si los filtros son correctos y existe información, el sistema presenta otra pantalla en la que se visualiza el mapa de Manizales con los gráficos de barras y el porcentaje de cada comuna de los indicadores o filtros consultados; en otro gráfico de barras se visualiza la información socioeconómica; por ejemplo, estrato socioeconómico, nivel educativo y estado civil, entre otros, por cada comuna.

\section{Resultados}

En esta sección se muestran los resultados del trabajo de investigación y el desarrollo de las tres etapas de la metodología, al presentar los módulos del sistema: 
- Módulo de administración. Se compone de tres opciones necesarias para obtener la información que se requiere en la posterior consulta, cada uno con estructuras de datos y reglas de migración y negocio diferentes:

- El sistema cuenta con la opción de migración de información del archivo de Sivigila.

- El sistema cuenta con la opción de migración de información del archivo de Sisben.

- El sistema cuenta con la opción de migración de datos de estadística de cantidad de población por comunas de la ciudad.

- Módulo de consulta de indicadores. Permite realizar la consulta a tres indicadores del evento epidemiológico para la enfermedad de tuberculosis. Está desarrollado con sentencias de base de datos diferentes para cada uno de los indicadores, e igualmente se desarrollaron sentencias de base de datos para la información socioeconómica del módulo de Sisben y Estadística. Al final se presentan tres gráficos:

- Mapa en coroplético: permite identificar, por medio de rango de colores, la cantidad de personas registradas por comunas que tienen el evento dependiendo del filtro en la consulta.

- Gráfico de barras del porcentaje del indicador: permite identificar en el gráfico de barras el porcentaje de cada comuna que registra por evento, dependiendo del filtro seleccionado anteriormente.

- Gráfico de barras de cantidad de datos socioeconómicos: permite obtener en un gráfico de barras la cantidad de personas que pertenecen al indicador previamente seleccionado, a la comuna y al filtro socioeconómico (estrato, nivel educativo, estado civil y tipo de vivienda). Esto con el fin de identificar las condiciones socioeconómicas de las personas afectadas.

- Módulo de consulta de filtros. Este módulo permite hacer consulta por género y por el rango de edad de las personas afectadas por el evento de la enfermedad de tuberculosis, así como mostrar los resultados a nivel de gráficos. Para estos filtros se realizaron consultas acordes con lo que se requería, y se toma la información de la misma estructura de datos de la consulta de indicadores.

\subsection{Resultados generales}

- El sistema presenta un mapa con datos de conteo de personas afectadas por una enfermedad para un indicador o filtro en una comuna de Manizales.

- Visualiza un gráfico de barras con el dato estadístico del indicador o filtro por comuna.

- Muestra un gráfico de barras con el valor de la cantidad de personas pertenecientes a una variable socioeconómica por comuna, con respecto al indicador o filtro consultado.

- La información es de valor importante para el epidemiólogo, ya que a partir de los resultados presentados por el sistema él, o el usuario del sistema, realiza un análisis de la situación de cada comuna con respecto a la cantidad de personas afectadas en toda la ciudad.

- El epidemiólogo queda satisfecho con el sistema y plantea la idea de "evolucionarlo", término indicado hacia la expansión de esta con la adición de nuevas funcionalidades, nuevas enfermedades, mayores detalles de las personas, entre otras que se expresan en el área de mejoras futuras.

- Las pruebas realizadas a cada subsistema con información correcta o mal diligenciada presentaron que el sistema realiza los cálculos como deben ser, solo si la calidad de la información es la correcta. En el caso de que la información no sea la correcta, el sistema permite identificar las columnas en las que se diligenciaron de forma no adecuada los registros, mostrando al usuario un mensaje que notifica los problemas presentados.

\section{Discusión de resultados y conclusiones}

\subsection{Discusión de resultado}

En la tabla 2 se hace un cuadro comparativo de los sistemas presentados en la sección de "Antecedentes", con los trabajos investigados más importantes que influyeron en la realización del Sistema para la Visualización en Mapas de Indicadores Epidemiológicos de Tuberculosis para La Secretaría de Salud de Manizales, en el que se 
tienen en cuenta tres factores importantes extraídos y analizados por cada trabajo. A continuación, se explica el significado de cada columna:

- Antecedentes: nombre del trabajo investigado en el apartado de "Antecedentes".

- Clasificación: para el caso de los trabajos investigados, se realizó una clasificación dependiendo del tipo de software desarrollado por cada uno. Para este caso, se encontraron tres tipos:

- Sistema georreferenciado: el trabajo investigado utiliza mapas digitales para ubicar geográficamente la información.

- Estadístico: el trabajo permite, mediante métodos estadísticos, calcular medias, cuantiles y cuartiles, entre otros.

- Epidemiológico: el trabajo permite ingresar información relacionada con las enfermedades epidemiológicas.

- Características: se enuncian las características del antecedente y se clasifican en dos:

- Tomadas para el sistema: características que se tomaron para el presente trabajo.

- Justificación del por qué no se tomó: se enuncia el por qué no se utilizó, y se da una justificación.

- Tipo de licencia: se enuncia el tipo de licencia para cada software resultante de la investigación correspondiente.

Como se puede observar en el cuadro comparativo, solo se tomó información para el presente trabajo de dos investigaciones realizadas que aportaron funcionalidades para la realización del sistema:

- SIG-EPI de la Organización Panamericana de la Salud. Sirvió para describir espacialmente los eventos de salud a partir de mapas temáticos, ya que permite la ubicación de la cantidad de personas que presentan tuberculosis en una comuna específica de la ciudad. De esta herramienta se tomó la forma en que se presentan los datos de las personas afectadas por una enfermedad en un lugar específico. En este caso, sería Manizales, dividida en sus comunas y presentadas con un color específico, dependiendo de la cantidad de personas afectadas por tuberculosis.

- Sistema gráfico georreferenciado de indicadores de género. De este trabajo se tomó la forma en que se clasifican los filtros para buscar información de las personas afectadas por alguna enfermedad epidemiológica, permitiendo conocer el género y el rango deedad. Además, sehace uso delainformación socioeconómica del afectado. También de este trabajo se extrae la forma de abordar la información de entrada para la consulta solicitada, eligiendo el tipo de filtro, bien sea por rango de edad específico (por ejemplo, buscar personas entre 15 y 30 años), o por género (masculino o femenino). Luego de elegir los filtros, la herramienta ubica la información en el mapa y se presenta un segundo filtro que permite detallar la información mediante los gráficos de barras, en el que se encuentra una nueva entrada de datos: se escoge si se desea ver información relacionada con el estrato socioeconómico (estrato 1,2 , etc.), nivel educativo (primaria, secundaria, profesional), estado civil (soltero, casado, entre otros), o tipo de vivienda (arrendada, propia, familiar).

Los demás trabajos mencionados en la sección de "Antecedentes" sirvieron como sustento teórico en el desarrollo del trabajo investigativo y en la realización del mismo:

- En el trabajo denominado Análisis de distribución de patrones territoriales en casos de tuberculosis, se tomó la forma en que se identifican los grupos de casos de tuberculosis, anexando una base teórica en la sustentación de los objetivos del trabajo y en el cumplimiento de la validación de los resultados.

- Para el otro trabajo, Los sistemas de información geográfica como herramienta para monitorear las desigualdades de salud, se toma de este información relacionada con los factores económicos y su determinación en la presencia de muertes por una enfermedad específica, dando como resultado la forma como afecta la tuberculosis a personas de estratos bajos y con un nivel de educación muy básico, o en ocasiones nulo. Al contar con la herramienta final, se logran identificar las zonas donde la Secretaría de Salud de Manizales debe estar alerta y pendiente, a fin de evitar las muertes innecesarias a causa de la tuberculosis en la ciudad.

En cuanto a las herramientas utilizadas en el desarrollo del proyecto, estas se escogieron gracias a las sugerencias realizadas por el contratista del área de Planeación y sig de la Alcaldía de Manizales, el ingeniero Mauricio Zuluaga, quien aportó los mapas y su visualización de la cartografía de la ciudad. El 
Tabla 2. Cuadro comparativo de sistemas de información similares

\begin{tabular}{|c|c|c|c|c|c|c|}
\hline \multirow[t]{2}{*}{ Antecedente } & \multicolumn{3}{|c|}{ Clasificación } & \multicolumn{2}{|c|}{ Características } & \multirow{2}{*}{$\begin{array}{l}\text { Tipo de } \\
\text { licencia }\end{array}$} \\
\hline & SIG & Estadístico & Epidemiológico & Tomadas para el sistema & $\begin{array}{l}\text { Justificación del } \\
\text { porque no se tomó }\end{array}$ & \\
\hline $\begin{array}{l}\text { SIG-EPI de la } \\
\text { Organización } \\
\text { Panamericana } \\
\text { de Salud }\end{array}$ & $\mathrm{x}$ & & & $\begin{array}{l}\text { Descripción espacial de } \\
\text { eventos de salud a partir } \\
\text { de mapas temáticos. }\end{array}$ & $\mathrm{N} / \mathrm{A}$ & Gratuita \\
\hline Epidat & & $\mathrm{x}$ & $\mathrm{x}$ & $\mathrm{N} / \mathrm{A}$ & $\begin{array}{l}\text { El sistema tiene } \\
\text { otro enfoque, es } \\
\text { más estadístico y no } \\
\text { maneja mapas. }\end{array}$ & Gratuita \\
\hline $\begin{array}{l}\text { Software } \\
\text { Centers } \\
\text { for Disease } \\
\text { Control and } \\
\text { Prevention }\end{array}$ & $\mathrm{x}$ & $\mathrm{x}$ & $\mathrm{x}$ & $\mathrm{N} / \mathrm{A}$ & $\begin{array}{l}\text { Requiere de pro- } \\
\text { cesos diferentes a } \\
\text { los planteados en } \\
\text { el presente trabajo, } \\
\text { además se nece- } \\
\text { sita información } \\
\text { adicional que no } \\
\text { se maneja en la } \\
\text { Secretaría de Salud } \\
\text { de Manizales. }\end{array}$ & Gratuita \\
\hline $\begin{array}{l}\text { Sistema grá- } \\
\text { fico georrefe- } \\
\text { renciado de } \\
\text { indicadores de } \\
\text { género }\end{array}$ & $\mathrm{x}$ & & & $\begin{array}{l}\text { Clasificación de los } \\
\text { filtros para las consultas } \\
\text { realizadas. } \\
\text { Referencia para la utili- } \\
\text { zación de información } \\
\text { socioeconómica. }\end{array}$ & $\begin{array}{l}\text { El sistema está dise- } \\
\text { ñado y parametriza- } \\
\text { do específicamente } \\
\text { para las necesidades } \\
\text { de México, sus } \\
\text { aspectos socioeco- } \\
\text { nómicos. }\end{array}$ & $\begin{array}{l}\text { Gratuita } \\
\text { (con } \\
\text { restric- } \\
\text { ciones) }\end{array}$ \\
\hline
\end{tabular}

Fuente: elaboración propia

ingeniero recomendó el uso de Open Street Map y Leaflet, debido a su experiencia en el área de SIG.

Por otra parte, el asesor de tecnología de la Secretaría de Salud recomendó para el desarrollo web, utilizar YII (framework para el lenguaje PHP) y MYSQL (como motor de base de datos). De acuerdo con las consultas realizadas en el apartado de la arquitectura, se identificó cómo para los gráficos de barras la librería que ofrece Googlechart sería una buena opción por ser esta herramienta sencilla, amigable y fácil de entender e implementar.

Para el caso de la arquitectura utilizada, el sistema se desarrolló como una aplicación web, ya que se requería un sistema listo para ser consultado sin estar sujeto a características de un equipo o requerimientos mínimos de sistemas o de instalación, asimismo, era necesario que pudiese ser consultado en un equipo sin depender de la ubicación del usuario del sistema y con un módulo de autenticación que permitiera resguardar la información de personas ajenas a la Secretaría de Salud, por medio de la autenticación con un usuario y una contraseña.

Se eligió que manejara tres capas en busca de una mejor separación de los objetivos y de las funciones que le corresponde a cada una (mantenibilidad, escalabilidad), y así definir las responsabilidades de cada una, de manera que resulte una estructura que permita realizar pruebas de manera sencilla y, además, la adición de nuevos módulos a fin de ampliar su funcionalidad (mejoras futuras).

Las capas son las siguientes y cada una fue explicada en el apartado de "Desarrollo en la arquitectura": 
- Capa de presentación

- Capa de lógica de negocio

- Capa de datos

La aplicación de las prácticas de Openup en el proyecto fue una opción indicada, ya que permite obtener una serie de documentos o resultados en la construcción del mismo.

Se incluyen unos vistos buenos que derivan del cumplimiento de cada uno de los objetivos del trabajo investigativo:

- La información es de valor importante para el epidemiólogo, al permitir, a partir de los resultados presentados por el sistema, realizar un análisis de la situación de cada comuna con respecto a la cantidad de personas afectadas en toda la ciudad.

- El epidemiólogo queda satisfecho con el sistema y plantea la idea de ampliarlo, término indicado para la expansión del sistema, adicionando nuevas funcionalidades, enfermedades y detalles de las personas, entre otras mejoras.

- Las pruebas realizadas a cada subsistema con información correcta o mal diligenciada demostraron que el sistema realiza los cálculos como deben ser solo si la calidad de la información es la correcta. En el caso contrario, el sistema permite identificar dónde están las inconsistencias.

Por último, se exponen las recomendaciones futuras más relevantes para que este proyecto pueda seguir aportando, tanto a nivel de la entidad, como a nivel académico.

- Se evidenció la necesidad de mejorar el proceso de registro de barrios en el sistema de Sivigila, para así garantizar la calidad de la información y los procesos estadísticos posteriores al registro, tanto en el sistema Sivigila, como en el sistema Indepi desarrollado. Por lo tanto, se recomienda realizar un módulo de barrios en el que se listen y estos no sean digitados, y así minimizar errores.

- Continuar este mismo proyecto, pero incluir comparaciones de datos con otros años con el propósito de percibir la diferencia año a año y el comportamiento del evento de la enfermedad de tuberculosis en la ciudad.
- Ampliar el alcance del proyecto para otros eventos epidemiológicos. Esto podría ser otro trabajo de grado en el que se incluya otra estructura de evento epidemiológico.

- La Secretaría de Salud le debe dar difusión al proyecto para que otras dependencias de la alcaldía adopten este tipo de trabajo, ya que pueden ser de gran utilidad en cualquier área que se plantee.

\subsection{Conclusiones}

Se obtuvo la aplicación planteada con los objetivos del proyecto, suministrando un sistema que permite consultar los indicadores epidemiológicos del evento de la enfermedad de tuberculosis, al reflejar la información en un mapa de la ciudad con una división en comunas en el que se logra percibir la cantidad de personas afectadas, el porcentaje en un gráfico de barras del indicador seleccionado y la cantidad de personas por consulta socioeconómica.

Con este proyecto se puede observar o concluir que si se aplican herramientas SIG a los datos de salud, se logra obtener información importante para el análisis de las enfermedades en la población, permitiendo visualizar la concentración de una enfermedad en específico - para este caso la tuberculosis-, en un área geográfica, identificando así zonas propensas al contagio. Esto ayuda a plantear soluciones eficaces que mitiguen la propagación de dicha enfermedad entre las personas.

Es una herramienta que sirve de apoyo analítico y puede llegar a generar conocimiento entre las entidades que la utilicen, permitiendo reconocer los patrones de la enfermedad y el comportamiento de la misma en la población. Se obtiene así un tiempo de respuesta rápido y de mayor exactitud, y se sabe de antemano cómo actuar ante determinado evento que se llegue a presentar.

Asimismo, permite ingresar datos de diferentes años para posteriormente consultar el comportamiento de los indicadores, observarlos en un mapa y analizarlos frente a otras épocas. De esta manera, construye un conocimiento firme frente a la enfermedad y relaciona la información socioeconómica de las personas, lo que permite identificar cuál es la población más vulnerable y realizar campañas que mitiguen el impacto de las enfermedades. 


\section{Referencias}

[1] Organización Mundial de la Salud, Epidemiología. Disponible en:http://www.who.int/topics/epidemiology/es/

[2] Constitución Política de Colombia, Art. 6. Julio 7 de 1991. Disponible en: http://www.ramajudicial. gov.co/documents/10228/1547471/CONSTITUCION-Interiores.pdf/8b580886-d987-4668-a7a8$53 \mathrm{f} 026 \mathrm{f} 0 \mathrm{f} 3 \mathrm{a} 2$

[3] Secretaría de Salud Pública de Manizales, Quienes Somos. Disponible en: http://manizalessalud.com/ quienes-somos/la-secretaria-de-salud/

[4] Secretaría de Salud Pública de Manizales, Planeación y Epidemiología. Disponible en: http://manizalessalud. com/quienes-somos/programas/planeacion-y-epidemiologia/

[5] Instituto Nacional De Salud, Manual para Análisis, Bogotá. Disponible en: http://www.saludputumayo. gov.co/documentos/NORMAS/MANUAL_INDICADORES.pdf

[6] Departamento Nacional de Planeación, Qué es el Sisben, Bogotá. Disponible en: https://www.sisben. gov.co/Informaci\%C3\%B3n/ ElSisb\%C3\%A9n.as px \#. VzJ-IIThDIU

[7] Organización Panamericana de la Salud, Aplicaciones y cooperación técnica. Disponible en: http://ais.paho. org/sigepi/index.asp?xml=aplica.htm

[8] Xunta de Galicia Consellería de Sanidade, Epidat. Disponible en: http://www.sergas.es/Saude-publica/ EPIDAT?idioma $=$ es

[9] Centers of Disease Control and Prevention, Epi info, Disponible en: http://wwwn.cdc.gov/epiinfo/

[10] Cámara de Diputados, Sistemas de consulta. Disponible en: http://www3.diputados.gob.mx/camara/001_diputados/006_centros_de_estudio/05_centro_de_estudios_ para_el_logro_de_la_igualdad_de_genero/01b_que_ hacemos/00e_sistemas_de_consulta

[11] Sistema gráfico georreferenciado de indicadores de género, CEAMEG. Disponible en: http://archivos.
diputados.gob.mx/Centros_Estudio/ceameg/informacion_analitica_2010/Doc_33.pdf

[12] Revista Geográfica de Valparaíso, "Análisis de distribución de patrones territoriales". Disponible en: http:// geografiapucv.cl/wp-content/uploads/ 2016/ 05/45_4.pdf

[13] G. Restrepo, J. Gonzales, Biometría Comunitaria, Fundación Universitaria Juan N. Corpas, Bogotá, 2012.

[14] Ministerio de Salud y Protección Social, Qué es Tuberculosis тв. Disponible en: http://www.minsalud. gov.co/salud/Paginas/Tuberculosis.aspx

[15] Organización Mundial de la Salud, Determinantes sociales de la salud. Disponible en: http://www.who. int/social_deter-minants/es/

[16] M. Villar-Aguirre, "Factores determinantes de la salud: Importancia de la prevención", Acta Médica Peruana, vol. 28, n. ${ }^{\circ} 24$, pp. 237-241, 2011. Disponible en: http:// www.redalyc.org/articulo.oa?id=96621053011

[17] L. S. Álvarez-Castaño, "Los determinantes sociales de la salud: más allá de los factores de riesgo", Gerenc. Polit. Salud, vol. 8, n.o 17, pp. 69-79, 2009. Disponible en: http://www.scielo.org.co/pdf/rgps/v8n17/v8n17a05.pdf

[18] Organización Panamericana de la Salud, Determinantes sociales en salud, Publicaciones Organización Panamericana de Salud Argentina, 2008. Disponible en: http:// publicaciones.ops.org.ar/publicaciones/piezas\%20comunicacionale s/curs oDDS/cursoesp/unidad1B.html

[19] J. Peña-Llopis, Sistema de Información Geográfica aplicadas a la gestión del territorio, Editorial Club Universitario. Disponible en: http://www.editorial-club-universitario.es/pdf/557.pdf

[20] J. Palomar-Vázquez, Introducción a gvSIG, Georreferenciación. Disponible en: http://personales.upv.es/ jpalomav/cursos/gvsig/gvsig_19.html? t6612.html

[21] ARCGIs-Resources, Georreferenciación y sistemas de coordenadas. Disponible en: http://resources.arcgis.com/es/help/getting-started/articles/ 026n0000000s000000.htm\%20

[22] Eclipse.org, Introduction to Openup (Open Unified Process), 2007. Disponible en: https://www.eclipse. org/epf/general/OpenUP.pdf 